\title{
La formación en Medicina Interna en Colombia
}

\section{Training in Internal Medicine in Colombia}

\author{
JaIRo H. RoA • BogotÁ, D.C.
}

Desde el establecimiento de los primeros programas de residencia en Medicina Interna en el país, la formación ha sido conjunta entre las universidades y los hospitales universitarios, de hecho en la década de los setenta cuando existió el sistema nacional de salud, la formación en Medicina Interna se hacía en hospitales universitarios y fueron estos últimos los que llevaron la carga de la formación, de hecho históricamente quien necesita especialistas son los pacientes y por lo tanto el sistema de salud tiene que encargase de formarlos. Con la reforma educativa de 1980 la formación en especialidades médico-quirúrgicas quedaron bajo el control directo de las universidades a través de sus facultades de medicina. Nunca se hizo un estudio nacional para establecer la verdadera magnitud del esfuerzo en la formación en Medicina Interna y las verdaderas relaciones entre los actores de la formación: residentes, profesores, facultades de medicina y hospitales universitarios.

En esta edición de Acta Médica Colombiana se publica el artículo Perfil de Formación del Posgrado en Medicina Interna en Colombia. Investigación realizada por el Comité de Educación (2004-2006) de la Asociación Colombiana de Medicina Interna, por los Doctores J.C. Velásquez, A.E. Pinilla, A. Pinzón, D. Severiche, R.J. Dennis, M.N. Niño de Arboleda (1). Es el primer esfuerzo para describir la formación en Medicina Interna en Colombia, esta encuesta fue contestada por la mayoría de los programas de formación en Medicina Interna en el país, y se llega a conclusiones muy interesantes que podíamos intuir, la mayoría de los hospitales universitarios no tienen una relación directa con las universidades, situación muy similar a la encontrada por Flexner en la primera década del siglo XX en los Estados Unidos, esto hace que haya una dicotomía entre el propósito de educación e investigación que debe caracterizar a un hospital universitario y una IPS tratando de sobrevivir en un sistema de salud muy adverso.

No es extraño encontrar que la mayoría de los docentes no tienen vinculación con la universidades, esto crea dudas sobre el verdadero papel de la universidad en la formación avanzada de posgrado, siendo muy claro que los docentes de un programa de residencia se encuentran es en el hospital universitario y no en las aulas de la universidad.

En cuanto a la formación es claro el énfasis actual en la formación de "internistas hospitalistas", los residentes realizan la mayoría de sus rotaciones en hospitales de tercer y cuarto nivel y muy poco tiempo en cuidado ambulatorio.

La investigación en los posgrados no es la mejor, aunque se ha observado en eventos nacionales como el Congreso Colombiano de Medicina Interna, el gran incremento de la presentación de trabajos científicos realizados por residentes.

Es evidente que el país necesita más internistas, los programas deberán aumentar sus cupos sin disminuir la calidad en la formación.

Tenemos un reto muy importante para este siglo, aumentar las relaciones entre el hospital universitario y las facultades de medicina, esto redundará en un cuerpo docente entusiasmado y comprometido con el esfuerzo educativo de las facultades de medicina, pero al mismo tiempo hará que los hospitales universitarios sean verdaderos ejemplos de formación e investigación con un excelente uso de los recursos.

Se deberá diversificar en algo la formación de los internistas generales, si bien hay mucha demanda para internistas que van a pasar la mayoría del tiempo en hospitales, ya sea en los Servicios de Medicina Interna, las Unidades de Cuidado Intensivo y los Servicios de Urgencias. Hay una gran necesidad de internistas en Cuidado Ambulatorio, el especialista
Dr. Jairo H. Roa: MD, M.Sc.Ed. FACP. Presidente Asociación Colombiana de Medicina Interna. Director Programa de Residencia Universidad del Bosque-Fundación Santa Fe de Bogotá. Bogotá, D.C. E-mail: jairoroamd@etb.net.co Recibido: 03/V/08 Aceptado: 04/V/08 
que atiende a los adultos es el internista y por lo tanto deberían tener una formación muy importante no solo en realizar historias clínicas perfectas, una de sus actividades básicas, sino en el uso adecuado de los recursos, conocimientos claros de prevención de la enfermedad y promoción de la salud. Es así como se hace necesario que los residentes de Medicina Interna utilicen un tiempo importante para el cuidado continuado de pacientes ambulatorios, esto les dará la experiencia necesaria para el trabajo ambulatorio donde son muy necesarios.

La realización de estudios como el presentado en esta edición de Acta Médica Colombiana es muy importante ya que nos da una información clara acerca de la formación en Medicina Interna y nos indica los cambios que debemos hacer en el futuro.

\section{Referencia}

1. Velásquez JC, Pinilla AE, Pinzón A, Severiche D, Dennis RJ, Niño de Arboleda MN. Perfil de formación del posgrado en Medicina Interna en Colombia. Investigación realizada por el Comité de Educación (2004-2006) de la Asociación Colombiana de Medicina Interna. Acta Med Colomb 2008; 33: 45-57. 